\title{
The Tev 2000 Report: Electroweak Physics with High Luminosity at the Fermilab Tevatron
}

\author{
Dan Amidei \\ Randall Laboratory of Physics \\ University of Michigan, \\ Ann Arbor, MI, 48109-1120 USA
}

\begin{abstract}
The addition of the Main Injector and the Antiproton Recycler to the Fermilab accelerator complex create the prospect of integrating samples in excess of $20 \mathrm{fb}^{-1}$ at the Tevatron collider. The Tev2000 report is a study of the "High$\mathrm{P}_{\mathrm{I}}$ " physics that may be realized with such data sets. We find a rich catalog of measurements, including characterization of the top quark, sensitivity to a light Higgs boson and low energy SUSY, and a precision electroweak program with sensitivity comparable and complementary to that of LEP-I.
\end{abstract}

\section{INTRODUCTION}

For the next decade, the Fermilab Tevatron Collider remains the high energy frontier of particle physics. Luminosity enhancements planned or possible with the Main Injector will dramatically increase the discovery reach, and, in conjunction with upgrades to the collider detectors, will move the experimental program into a regime of precision hadron collider physics.

The Tev2000 Working group has completed a preliminary study of the physics potential for an extended Tevatron program, and this paper is a summary of that work [1]. I concentrate here on top physics, a completely new arena for particle physics at an existing U.S. facility. I also include brief summaries of the prospects for detecting a light Higgs boson or low energy SUSY, as well as the outline of a global precision electroweak program.

In the Tev2000 report, we avoid the vagaries of operating point or schedule, and simply specify the physics reach as a function of integrated luminosity, assuming detector performance maintained at the level of the CDF and DØ upgrades for Tevatron Run 2, beginning in 1999. This summary presents expectations for 2 luminosity goals: the present Run 2 plan of $1-2 \mathrm{fb}^{-1}$, and a Run 3 of greater than $25 \mathrm{fb}^{-1}$. 


\section{TOP PHYSICS}

The top quark is strongly coupled to the electroweak symmetry breaking sector, and decays into a $b$ quark and a real $W$ before hadronizing. The top quark is an obvious new avenue for study of the fermion sector, and offers a number of experimental techniques not previously available for quarks.

\section{A Event Samples in $t \bar{t}$}

The standard t $\bar{t}$ selection is based on the expected decay chain $t \bar{t} \rightarrow$ $\left(W^{+} b\right)\left(W^{-} \bar{b}\right)$ and the subsequent decays of the W's into fermion pairs [2]. At least one $W$ is tagged in the mode $W \rightarrow l \nu$ by requiring an isolated high lepton (e or $\mu$ ) with large "transverse momentum" $\left(\mathrm{P}_{T}\right)$ to the beamline and large "missing transverse energy", $\mathbb{E}_{T}$. In the "dilepton" analysis the leptonic decay of the other $W$ is identified with a loose lepton selection; this mode has small backgrounds but small branching fraction of just $4 / 81$.

In the "lepton+jets" mode, the second $W$ decays to quark pairs, giving larger branching fraction of $24 / 81 \approx 30 \%$ (lepton $=\mathrm{e}$ or $\mu$ ). The final state of $(1 \nu \mathrm{b})(\mathrm{jjb})$ is separated from the primary background, $W+$ jets, by requiring a large multiplicity of high $E_{T}$ jets and also evidence of a $B$ decay, using either secondary vertex identification (SVX) or a tag of the "soft lepton" from $\mathrm{b} \rightarrow \mathrm{l} \nu_{l} \mathrm{X}$ (SLT). The largest sample results from requiring at least 3 jets with $\mathrm{E}_{T} \geq 15 \mathrm{GeV}$ and $|\eta| \leq 2.4$. The constrained fit technique used in the top mass measurement requires a "completely reconstructable" event having at least 4 final state jets.

Future $t \bar{t}$ event yields at the Tevatron can be inferred with reliable precision by extrapolating from the presently understood situation. The Upgrades for Run 2 will have improved lepton acceptance and much improved b-tagging. The efficiency for tagging at least $1 \mathrm{~b}$ will be $85 \%$ per event, and the efficiency for tagging both b's will be $42 \%$ per event. We will assume that selection effciencies can be maintained at high luminosity by staged evolution in detector technologies. The total efficiency including branching fraction $\epsilon \cdot B$ for some pertinent final states is shown in Table 1.

We calculate absolute yields at the Run II operating point of $\sqrt{s}=2.0 \mathrm{TeV}$, using $\sigma_{t \vec{t}}=6.8 \mathrm{pb}$ for $\mathrm{m}_{\mathrm{t}}=175 \mathrm{GeV} / \mathrm{c}^{2} \quad[3]$. The future yields are shown for the benchmark data sets in Table 1 . At $\mathrm{m}_{\mathrm{t}}=175 \mathrm{GeV} / \mathrm{c}^{2}$ each inverse femtobarn at the Tevatron will yield approximately 600 identified, b-tagged events and approximately 250 double b-tagged, completely reconstructable events. 
TABLE 1. Top yields

\begin{tabular}{lccc}
\hline & & & \\
\hline Mode & $\epsilon \cdot B$ & $1 \mathrm{fb}^{-1}$ & $10 \mathrm{fb}^{-1}$ \\
\hline produced & & $6.8 \mathrm{~K}$ & $68 \mathrm{~K}$ \\
dilepton & $1.1 \%$ & 82 & 820 \\
$W+3 \mathrm{j}$ & $10 \%$ & 680 & $6.8 \mathrm{~K}$ \\
$W+3 \mathrm{j} * \mathrm{~b}$ & $8.6 \%$ & 584 & $5.8 \mathrm{~K}$ \\
$\mathrm{~W}+4 \mathrm{j}$ & $8.9 \%$ & 605 & $6.0 \mathrm{~K}$ \\
$\mathrm{~W}+4 \mathrm{j} * \mathrm{~b}$ & $7.6 \%$ & 517 & $5.2 \mathrm{~K}$ \\
$\mathrm{~W}+4 \mathrm{j} * \mathrm{bb}$ & $3.8 \%$ & 258 & $2.6 \mathrm{~K}$ \\
\hline \hline
\end{tabular}

\section{B Measurement of the Top Mass}

The mass of the top quark is a fundamental Standard Model parameter and should be measured as accurately as possible. The best understood mass measurement technique at present is complete reconstruction in $t \overline{\mathrm{t}} \rightarrow$ $W+4$ jets [4]. Events with at least four jets are selected as above, and the lepton and the four highest $\mathrm{E}_{T}$ jets in the event are fit to the hypothesis $t \overline{\mathrm{t}} \rightarrow(W b)(W b) \rightarrow(l \nu b)(j j b)$. Each jet is extrapolated back to a parton en ergy by correcting on average for instrumental and QCD effects. The 2-C fit has multiple solutions in each event, and the configuration with lowest $\chi^{2}$ and consistency with b-tagging is chosen. The shape of the mass spectra for various values of $m_{t}$, as well as for the expected backgrounds, are derived from Monte Carlo samples, and a maximum likelihood fit to the data spectrum yields the best estimate of the top mass.

Almost all of the systematic uncertainties in the top mass measurement are coupled to the reliability of the Monte Carlo models for the spectrum of fit masses in signal and background. Assuming the theory model is accurate, most of the uncertainty concerns resolution effects. Instrumental contributions include calorimeter nonlinearity, losses in cracks and dead zones, and absolute energy scale. A larger and more intractable part of the energy resolution concerns the reliability of the extrapolation to parton energies, and it is the understanding of QCD, not the detector, which limits the mass resolution. All of these issues can be addressed by in situ calibration procedures such as $E_{T}$ balancing in $Z / \gamma+1$ jet events and isolation of $W \rightarrow j j$ in top events.

The statistical error for the three event classes $W+4 j, W+4 j+b$, and $\mathrm{W}+4 \mathrm{j}+\mathrm{bb}$ are shown in Table 2 , as understood from the present measurement, and extrapolated to the future scenarios assuming the event yields above. Note that in large samples the double tagged events have good statistical precision.

We have also made a careful accounting of the probable evolution of the systematic uncertainty for the single tagged analysis. The in situ energy calibration techniques have been benchmarked with simulation studies for $1 \mathrm{fb}^{-1}$, 
TABLE 2. Expected $\mathrm{m}_{\mathrm{t}}$ precision, all entries in $\mathrm{GeV} / c^{2}$.

\begin{tabular}{lccc}
\hline \hline Mode & $\delta \mathrm{m}_{\mathrm{t}}$ & $1 \mathrm{fb}^{-1}$ & $10 f b^{-1}$ \\
\hline stat. $\mathrm{W}+4 \mathrm{j}$ & $38 / \sqrt{N}$ & 1.6 & 0.5 \\
stat. $\mathrm{W}+4 \mathrm{j}+\mathrm{b}$ & $35 / \sqrt{N}$ & 1.5 & 0.5 \\
stat. $\mathrm{W}+4 \mathrm{j}+\mathrm{bb}$ & $27 / \sqrt{N}$ & 1.7 & 0.5 \\
\hline sys. $\mathrm{W}+4 \mathrm{j}+\mathrm{b}$ & see text & 3.7 & 1.2 \\
\hline total $\mathrm{W}+4 \mathrm{j}+\mathrm{b}$ & $\delta_{\text {stat }}^{2}+\delta_{\text {sss }}^{2}$ & 4.0 & 1.3 \\
\hline
\end{tabular}

and the resolution improvement from these is slightly worse than $\sqrt{N}$. The b-tag bias can be addressed in control sample studies, and should scale as $1 / \sqrt{N}$. Control of the small but significant uncertainty due to background modelling can be done in a large $\mathrm{Z}+$ jets sample, and this has also been benchmarked for $1 \mathrm{fb}^{-1}$. The net effect of this more careful consideration of the systematic error is listed in the fourth row of Table 2, and, compared to the row above, is seen to be slightly degraded from simple $1 \sqrt{N}$ scaling.

Adding these systematic errors in quadrature with the statistical error leads to the top mass precision listed at the bottom of Table 2 . With $10 \mathrm{fb}^{-1}$ at the Tevatron, the experimental contributions to the top mass uncertainty will be limited to the order of $1.3 \mathrm{GeV} / c^{2}$ per experiment.

Other techniques under study include likelihood fits to the kinematics in the dilepton sample, complete reconstruction in the 6 jet mode, and accurate measurement of the mean decay length in b's from top decay, and all of these techniques project to errors of $\sim 1-2 \mathrm{GeV}$ for $10 \mathrm{fb}^{-1}[5-7]$. Since these techniques are statistically independent, we believe that $10 \mathrm{fb}^{-1}$ will allow a combined measurement of the top mass with the control of the experimental uncertainties at the level of $1 \mathrm{GeV} / \mathrm{c}^{2}$.

Ultimately, the theoretical inputs must also be verified. For instance, the distribution of fit masses used as input templates to the likelihood fit are derived from a theoretical calculation, and several studies raise questions concerning the modelling of hard gluon radiation and other subtleties in the final state [8]. Resolution of these concerns requires more statistical precision than presently available, but we expect that the theoretical uncertainties will ultimately be controlled at the level of the experimental precision, of order 1 $\mathrm{GeV} / c^{2}$.

Taking all of the above into account, we believe that $10 \mathrm{fb}^{-1}$ at the Tevatron will allow a measurement of the top mass with a precision of 2 $\mathrm{GeV} / c^{2}$ per experiment.

If the $\mathrm{W}$ mass is known to $20 \mathrm{MeV} / \mathrm{c}^{2}$, a precision of $2 \mathrm{GeV}$ on the top mass will fix the Higgs mass to within $50 \%$ of itself [1]. Improvement beyond $2 \mathrm{GeV}$ in top mass precision does not affect the Higgs precision unless the $W$ 
mass precision is likewise improved.

\section{Top Pair Production}

An accurate measurement of the $t \bar{t}$ production cross section is a precision test of QCD. A cross section significantly higher than the theoretical expectation would be a sign of non-Standard Model production mechanisms, for example the decay of a heavy resonant state into $t \bar{t}$ pairs [9], or anomalous couplings in QCD [10].

Future samples will have large statistics in the $1+$ jets mode and systematic uncertainties will be the limiting factor.

For the acceptance, the reliability of jet counting and b-tagging are at issue. Initial state radiation can be examined using a sample of $Z+j e t s$, while the jet energy threshold uncertainty can be addressed as in the top mass discussion. With $1 \mathrm{fb}^{-1}$ of data it will be possible to measure the b-tagging efficiency in top events, using dilepton events selected without a b-tag and the ratio of single to double tags in lepton plus jets events. We assume that these studies will give uncertainties that scale with $\sqrt{N}$.

With large samples, one can measure the bottom and charm content as a function of jet multiplicity in $\mathrm{W}+$ jet events using the $\mathrm{c} \tau$ distribution of the tagged jets and use this to tune the Monte Carlo models for $\mathrm{W}+3$ or more jet backgrounds. Finally, in Run 2 and beyond, the luminosity will be measured either through the $W \rightarrow l \nu$ rate, or the mean number of interactions per crossing, and we will assume $5 \%$ for the future precision of the luminosity normalization.

Accounting for all effects we find that the total $t \tilde{t}$ cross section can be measured with a precision of $11 \%$ for $1 \mathrm{fb}^{-1}$ and $5.9 \%$ for $10 \mathrm{fb}^{-1}$. This will challenge QCD, and provide a sensitive test for non-Standard production mechanisms and decay width to $\mathrm{Wb}$.

\section{D "Single Top" Production}

At a hadron collider, the top quark decay width $\Gamma(t \rightarrow X)$ cannot be directly measured in the $t \bar{t}$ sample, but its main component can be accessed through single top processes [11]. The single top cross section is directly proportional to the partial width $\Gamma(t \rightarrow b+W)$ and, assuming there are no anomalous couplings, this is a direct measure of $\left|V_{t b}\right|^{2}$.

The principal processes leading to single top production are $q^{\prime} \bar{q} \rightarrow t \bar{b}$, via an $s$-channel " $W^{*}$ " and $q b \rightarrow q^{\prime} t$, via $t$-channel " $W$-gluon fusion". For $\mathrm{m}_{\mathrm{t}}=$ $175 \mathrm{GeV} / c^{2}, W$-gluon fusion, at $1.6 \mathrm{pb}$, is twice as large as $W^{*}$ at $0.8 \mathrm{pb}$, and the combined rate for single top production by these two processes, $\sim 2.4 \mathrm{pb}$, is only a factor of 3 down from the $t \bar{t}$ rate at this energy [12]. 
We have simulated the measurement of electroweak single top production using the ONETOP Monte Carlo with $\mathrm{m}_{\mathrm{t}}=170 \mathrm{GeV}$ [13]. We model detector performance for $b$ tagging and jet energy resolutions based on the top mass and cross section analyses described above. B tagging backgrounds are modelled on the current CDF analysis. Our data selection criteria were similar to the $t \bar{t}$ selection, except to ask for just 2 and only 2 jets, with at least one b-tag.

The signal for single top production is a peak in the $W b$ invariant mass plot. We find that the shape of the signal-plus-background curve is easily distinguished from the background shape alone. We calculate the fractional statistical uncertainty in the cross section as $\sqrt{S+B} / S$, where the size of signal $(S)$ and background $(B)$ are the numbers of each kind of event in the a mass peak window of $50 \mathrm{GeV}$ around the generated top quark mass. We find a signal yield of approximately 100 events per $\mathrm{fb}^{-1}$, above a background about twice as large, mainly from $W+b \bar{b}$. With $10 \mathrm{fb}^{-1}$ it will be possible to measure the single top cross section with a statistical precision of $5.5 \%$.

Many of the sources of systematic uncertainty in the single top cross section are common to the $t \bar{t}$ cross section discussed above. We assume that systematic uncertainties related to selection efficiencies and backgrounds will shrink as $\sqrt{N}$ and find that for large samples the dominant uncertainty is that of the luminosity normalization. For the case of $10 \mathrm{fb}^{-1}$ we find that the measurement of the single top cross section will have a total uncertainty of approximately $10 \%$.

We have made a detailed study of the extraction of $\Gamma(t \rightarrow b+W)$, and $\left|V_{t b}\right|^{2}$ from the combined single top cross section [14]. The constant of proportionality between the cross section and the width has theoretical uncertainties originating in $\alpha_{s}$, the parton distribution functions, and the choice of scale $Q^{2}$. These are estimated to total roughly $10 \%$ at present [15], and we assume that better measurements of parton distributions and $\sigma_{\mathrm{t}} \overline{\mathrm{t}}$ will improve this to $7 \%$. Combining all uncertainties, we find that a measurement of the inclusive single top cross section with $10 \mathrm{fb}^{-1}$ will yield the partial width $\Gamma(t \rightarrow W b)$ with precision of $12 \%$, and therefore $V_{t b}$ with a precision of $6 \%$.

\section{E Search for $t \bar{t}$ Resonances}

Several models have been proposed for extensions of the Standard Model which would produce enhancements or resonances in the $t \bar{t}$ invariant mass $\left(M_{t \bar{t}}\right)$ spectrum $[16,17]$.

We have studied sensitivity to this kind of object via direct search for resonant structure in the $M_{t i}$ distribution. We reconstruct $M_{t i}$ on an event-byevent basis using the same event sample and constrained fitting techniques used in the top mass measurement, with an additional constraint that the $t$ and $\bar{t}$ decay products have a mass equal to the measured $M_{\text {top }}$.

For definiteness, we use the example of a topcolor $Z^{\prime}$ decaying to a $t \bar{t}$ pair, 
assuming that the resonance width is less than the detector resolution on $M_{i \bar{t}}\left(\approx 6 \%\right.$ at $\left.M_{t i}=800 \mathrm{GeV} / \mathrm{c}^{2}\right)$. The cross section, $\sigma \cdot B(X \rightarrow t \bar{t})$, is determined by theory, and the Pythia Monte Carlo provides the decay $X \rightarrow t \bar{t}$. The acceptance including all BR's, detector, and selection effects is $6.5 \%$ and approximately flat vs. $M_{i t}$.

We add the distribution for a $Z^{\prime}\left(M_{Z^{\prime}}=800 \mathrm{GeV} / \mathrm{c}^{2}, \Gamma_{Z^{\prime}}=1.2 \%\right)$ to the $M_{t \bar{t}}$ distribution from standard model $t \bar{t}$ production, quantify the excess by fitting the $M_{t \bar{t}}$ distribution below the resonance to estimate the background in the region $700-900 \mathrm{GeV} / \mathrm{c}^{2}$. We define the discovery limit as the minimum $\sigma$. $B(X \rightarrow t \bar{t})$ for the production of $X \rightarrow t \bar{t}$ in order to observe a $\geq 5$ sigma excess. With $10 \mathrm{fb}^{-1}$, we will be able to observe a narrow $Z^{\prime}$ resonance decaying to $\mathrm{t}^{-}$ out to approximately $800 \mathrm{GeV}$.

\section{F Top Decay}

In the Standard Model with 3 generations, existing experimental constraints and the unitarity of the CKM matrix require $V_{t b} \simeq 1$, predicting that the weak decay of the top will proceed almost exclusively through $\mathrm{W}+\mathrm{b}$. The $t \rightarrow W b$ decay vertex is completely fixed by the universal $\mathrm{V}-\mathrm{A}$ coupling to the $\mathrm{SU}(2)$ bosons. For $m_{t}=175 \mathrm{GeV} / c^{2}$, the partial (but almost total) decay width is $\sim 1.8 \mathrm{GeV}$ [18], cutting off the long distance part of the strong interaction. There is no hadronization: all strong interaction issues for the top quark should be well described by perturbative QCD, and charge and helicity information should flow directly to the final state. The top is the first opportunity to study the decays of a naked quark, with experimental techniques and advantages familiar from muon decay.

The detailed experimental issues pertinent to the study of top decays are similar to the efficiency and background issues discussed above in relation to the cross section measurement, and the energy scale issues discussed above in relation to the mass measurement. The comments below are brief, and further detail can be found in the Tev2000 Report [1].

\section{The $W-t-b$ Vertex}

It is possible that the physics of an underlying theory at a high mass scale may couple to the large top mass, appearing as new non-universal top interactions [19]. One manifestation of anomalous couplings would be a departure from the predicted mixture of $W$ helicity states in the decay. Since the top decay precedes hadronization, the $W$ polarization information persists in the final state, and is experimentally accessible through the charged lepton helicity angle, $\cos \theta_{e}^{*}$, which is measured in the lab frame $[20]$ as 


$$
\cos \theta_{e}^{*} \approx \frac{2 M_{e b}^{2}}{m_{e b \nu}^{2}-M_{W}^{2}}-1
$$

A general analysis of the $\mathrm{W}$ helicity states through the $\cos \theta_{e}^{*}$ distribution can then be performed.

The Standard Model predicts only left-handed or longitudinal W's in top decay, with the branching fraction to the longitudinal component depending only on the top mass. For $m_{t}=170 \mathrm{GeV} / c^{2}, B F\left(t \rightarrow b+W_{\text {long }}\right)=69.2 \%$. Non-universal top couplings will, in many cases, appear as a departure of $B F\left(t \rightarrow b+W_{\text {long }}\right)$ from the value expected for the measured $m_{t}$.

We have studied $B r\left(t \rightarrow b+W_{\text {long }}\right)$ as a sensitivity benchmark for anomalous top couplings. We use a four vector level Monte Carlo [13] with selection bias and resolution smearing modeled on the CDF experience. We assume that the constrained mass fit will allow us to know perfectly which $b$ jet belongs to the semi-leptonic top decay. We correct the $\cos \theta_{e}^{*}$ distribution for the bias imposed by the selection cuts. We fit the distribution the Standard Model hypothesis for the admixture of $W_{\text {long }}$ and $W_{\text {left }}$ and get a good fit with $B F\left(t \rightarrow b+W_{\text {long }}\right)=0.708 \pm 0.030$, as shown.

After accounting for the effect of combinatorics, energy scale uncertainties, and backgrounds, we find that for sample sizes expected to be available at a high luminosity Tevatron, of order $10 \mathrm{~K}$ and above, the top quark decay branching fraction to longitudinal $\mathrm{W}$ bosons may be measured with a statistical precision approaching $1 \%$, and is systematically limited. We have also studied the effect of a $\mathrm{V}+\mathrm{A}$ term, and find that it would be discernible with similar sensitivity.

\section{Measurement of $a t \rightarrow b$ Branching Fraction}

In the Standard Model, a $176 \mathrm{GeV}$ top quark decays almost exclusively to b's. This is easy to test. One looks at top events containing W's, and measures the branching fraction into $b$ 's:

$$
B F(t \rightarrow(W) b)=\frac{\left|V_{t b}\right|^{2}}{\left|V_{t d}\right|^{2}+\left|V_{t s}\right|^{2}+\left|V_{t b}\right|^{2}}
$$

The notation is meant to remind that this is the fraction of top decays to W's that also contain b's. Since the standard analysis identifies $t \bar{t}$ events by requiring at least $1 \mathrm{~W}$ and $1 \mathrm{~b}, B F(t \rightarrow(W) b)$ is measured from the number and distribution of tagged $b$-jets in top events. There are three methods which can be employed:

- The ratio of double $b$-tagged to single $b$-tagged events in the $b$-tagged lepton plus jets sample.

- The number of $b$ tagged jets in the dilepton sample. 
The number of times that events tagged by both the secondary vertex and soft lepton algorithms have both tags in the same jet vs. the number of times the tags are in different jets.

These techniques are not exclusive, and can be combined. This analysis has been carned out in the present CDF data set, and the precision can be scaled to the expected size of the Run 2 samples. The systematic uncertainty is dominated by the uncertasnty on the tagging efficiency, which is measured from the data using $b$ semileptonic decays, and will fall as $1 / \sqrt{N}$, provided that the experiments can contınue to record these events. The small non$t \bar{t}$ backgrounds will be measured to high accuracy by Run 2. A sample of $10 \mathrm{fb}^{-1}$ at the Tevatron allows the measurement of the branching fraction $B F(t \rightarrow(W) b)$ with a precision of $1 \%$.

\section{Measurement of a $t \rightarrow W$ Branching Fraction}

If all top decays proceed through $\mathrm{W}$ emission, the ratio of dilepton to single lepton events is $R_{l}=1 / 6$. If $t$ decays include a non-W state with no leptonic decays, the branching fraction to W's is given in terms of the ratio $R_{l}$ as

$$
B F(t \rightarrow W(b))=\frac{9 R_{l}}{1+3 R_{l}}
$$

The expected precision on this ratio can be scaled from the present understanding of cross section measurements in the $1+$ jets and dilepton modes. We find that with $10 \mathrm{fb}^{-1}$ the ratio of dilepton to single lepton rates in top events will allow determination of the top branching fraction to W's in association with $b$ to a precision of $3.5 \%$.

This analysis is obviously model dependent, but consistent with the popular non-standard model that $t \rightarrow W b$ may be augmented with $t \rightarrow H^{+} b$ where in this case $B F\left(H^{+} \rightarrow c \bar{s}\right)=100 \%$. The $\mathrm{W}$ branching fraction limit can be turned around to give a limit on $t \rightarrow H^{+} b$; we find that $10 \mathrm{fb}^{-1}$ at the Tevatron will allow observation of a charged Higgs in top decay down to branchung fractions of $6 \%$. The limits attanable by $10-20 \mathrm{fb}^{-1}$ of $p \bar{p}$ data, in conjunction with the CLEO $B(b \rightarrow s \gamma)$ measurement will be enough to exclude $m\left(H^{+}\right) \leq \sim$ $m(t)$ for any value of $\tan \beta$

\section{Rare Top Decays to $W, Z$, and $\gamma$}

Standard Model predictions for the branchung fractions of FCNC decays are around $10^{-10},[21]$, so any observation of such decays will signal new physics. As illustration, we consider the signal for a flavor changing neutral current decay $t \rightarrow c \gamma$ in a $t \bar{t}$ event. If the other top in the event decays in the leptonic channel, the acceptance is almost the same as the standard model $1+$ jets 
TABLE 3. A Top Physics Program: Summary of expected precision vs integrated luminosity at the Tevatron

\begin{tabular}{|c|c|c|}
\hline Measurement & $1 \mathrm{fb}^{-1}$ & $10 \mathrm{fb}^{-1}$ \\
\hline \multicolumn{3}{|l|}{ Yields } \\
\hline$\overline{N_{3 j e t+b-t a g}}$ & 580 & $5.8 \mathrm{~K}$ \\
\hline $\mathrm{N}_{4 \mathrm{jet}+2 \mathrm{~b}-\mathrm{tag}}$ & 260 & $2.6 \mathrm{~K}$ \\
\hline$\delta \mathrm{m}_{\mathrm{t}}$ & 3.5 & 2.0 \\
\hline \multicolumn{3}{|l|}{ Production } \\
\hline$\overline{\delta \sigma_{t i}}$ & $11 \%$ & $6 \%$ \\
\hline$\delta \sigma_{t} / \sigma_{t+3}$ & $14 \%$ & $4.8 \%$ \\
\hline$\delta \sigma_{t \bar{b}}$ & $26 \%$ & $10 \%$ \\
\hline$\delta \sigma \cdot \mathrm{B}\left(\mathrm{Z}^{\prime} \rightarrow \mathrm{t} \overline{\mathrm{t}}\right)$ & $100 \mathrm{fb}$ & $25 \mathrm{fb}$ \\
\hline \multicolumn{3}{|l|}{ Decay } \\
\hline$\overline{\delta B F}(t \rightarrow(W) b)$ & $3 \%$ & $1.0 \%$ \\
\hline$\delta B F(t \rightarrow W(b))$ & $10 \%$ & $3.5 \%$ \\
\hline$\delta \mathrm{BF}\left(\mathrm{W}_{\mathrm{V}+\mathrm{A}}\right)$ & $2 \%$ & $0.6 \%$ \\
\hline$\delta \mathrm{BF}\left(\mathrm{W}_{\text {long }}\right)$ & $4 \%$ & $1.3 \%$ \\
\hline$\delta \Gamma(t \rightarrow W b)$ & $28 \%$ & $12 \%$ \\
\hline$\delta V_{t b}$ & $14 \%$ & $6 \%$ \\
\hline \multicolumn{3}{|l|}{ Rare Decays } \\
\hline$\overline{\mathrm{Br}(\mathrm{c}+\gamma)}$ & $3.0 \times 10^{-3}$ & $4.0 \times 10^{-4}$ \\
\hline $\mathrm{Br}(\mathrm{c}+\mathrm{Z})$ & $1.5 \times 10^{-2}$ & $3.8 \times 10^{-3}$ \\
\hline $\mathrm{Br}(\mathrm{Hb})$ & $15 \%$ & $6 \%$ \\
\hline
\end{tabular}

mode, and it then becomes a simple matter to scale from present results. The background from $W+\gamma+$ two jets is about $1 \mathrm{fb}$. Although it is unlikely that this background will be kinematically consistent with $t \bar{t}$ (for example, that $m(\gamma+j)=m(t))$, we take the very conservative assumption that this background is irreducible. We find that $10 \mathrm{fb}^{-1}$ will probe branching fractions for this decay down to $4.0 \times 10^{-4}$

Sensitivity to other rare decays can be scaled from this estimate. For the case $t \rightarrow Z+c$, where the $Z$ decays to leptons, after adjusting for branching ratios and different backgrounds, we find sensitivity down to $3.8 \times 10^{-3}$.

\section{G Conclusions for Top Physics}

We have reviewed the prospects for top physics at the Fermilab Tevatron in the Main Injector Era. The conclusions are preliminary, but have the strength of being extrapolations from real measurements in the well understood environments of the present day Collider experiments. We believe that this is only the beginning of the catalog of top physics measurements at the Teva- 
tron, and that this report is best interpreted as a survey of sensitivities in each of the categories of mass reconstruction, cross sections, branching ratios, decay dynamics, and rare decays. In the not unlikely event that this 180 $\mathrm{GeV} / c^{2}$ fermion harbors surprises, this study benchmarks the capability to explore the new physics at the Tevatron facility.

\section{OTHER HIGH $\mathrm{P}_{\mathrm{T}}$ PHYSICS OPPORTUNITIES}

In addition to the prospects for top physics, large integrated data sets at the Tevatron will allow incisive measurements in the electroweak sector, discovery of a Light Higgs boson, and a probe of a significant part of the parameter space of the Minimal Supersymmetric Model. These prospects are summarized briefly here, detailed discussion can be found in the Tev 2000 report.

\section{A Light Higgs Physics}

A light intermediate-mass scalar in the mass region $80 \mathrm{GeV} / \mathrm{c}^{2}<m_{H}<$ $130 \mathrm{GeV} / \mathrm{c}^{2}$ is predicted by minimal supersymmetric models, and current precision electroweak data also show a slight preference for a low mass Higgs. This study confirms recent theoretical speculation that there is a luminosity threshold for the detection of a light Higgs boson at the Tevatron, and suggests that this threshold varies from 5 to $25 \mathrm{fb}^{-1}$ as $m_{H}$ varies from 60 to $120 \mathrm{GeV} / \mathrm{c}^{2}$.

- The process $q \bar{q} \rightarrow W H$, with $H \rightarrow b \bar{b}$, is the best single mode for the detection of light Higgs boson at the Tevatron, and leads to the luminosity thresholds stated above. The analysis relies heavily on the understanding of b-tagging, the "W + flavor" backgrounds, and mass fitting with jets, and is therefore a natural complement and extension of the top physics program.

- The process $p^{+} p^{-} \rightarrow(W, Z) H$, with $H \rightarrow \tau^{+} \tau^{-}$and $(W, Z) \rightarrow j j$, is difficult at the Tevatron due to the large $\left(Z \rightarrow \tau^{+} \tau^{-}\right) j j$ background, but may add to the overall significance of the observation. Other channels, such as $\mathrm{ZH}$ with $\mathrm{Z} \rightarrow \nu \bar{\nu}$ and $H \rightarrow b \bar{b}$, have not been investigated, and should be. Because a set of combined channels is likely to have better significance than our single studied channel of WH with $H \rightarrow b \bar{b}$, the luminosity thresholds above are probably conservative, and the mass reach may be slightly higher.

- We have studied the potential of the $\mathrm{W}+H \rightarrow b \bar{b}$ measurement at the LHC, assuming equivalent detection efficiencies, etc and find that it is difficult there because of large top backgrounds. It may be that the intermediate mass region is accessible at the LHC only via the rare decay 
mode $H \rightarrow \gamma \gamma$. Since the branching fraction to $\gamma \gamma$ varies with the choice of SUSY parameters, the LHC cannot prove that the light Higgs boson of SUSY does not exist if it is not found there.

- The process $q \bar{q} \rightarrow W H$ is complementary to the LEP II/NLC process $q \bar{q} \rightarrow Z H$, since they involve the coupling of the Higgs boson to different weak bosons. The ratio of these couplings can vary in multi-Higgs models with multiplets other than doublets (e. g., Higgs triplets).

Although further study is needed, the opportunity to detect a light Higgs boson at the Fermilab Tevatron appears to be real.

\section{B Supersymmetric Physics}

Supersymmetry is an elegant and comprehensive extension to the Standard Model. It solves the gauge hierarchy problem, provides a candidate for cold dark matter, is consistent with gauge unification, and is naturally decoupled from Standard Model particles. It is a feature of most string theories.

The experimental consequence of supersymmetry at the weak scale is the presence of 32 new particles in the mass range of about 100 to $1000 \mathrm{GeV} / \mathrm{c}^{2}$. It is not surprising that none of these particle have been discovered yet, since current facilities are only sensitive below this mass range. However, with the increased luminosity available at TeV33, the Tevatron will be able to probe a significant fraction of the expected SUSY mass range for the first time.

Using a constrained minimal supersymmetric model (MSSM) with four free parameters and an unknown sign, a detector similar to the upgraded $D \emptyset / C D F$ detectors, and an integrated luminosity of $25 \mathrm{fb}^{-1}$ at $\mathrm{TeV} 33$, our preliminary conclusions are:

- We will be able to search for charginos with masses up to $250 \mathrm{GeV} / \mathrm{c}^{2}$. The mass reach depends on the exact value of the unknown SUSY parameters, but a significant fraction of the possible parameter space has light charginos accessible at the Tevatron.

- The Tevatron can find gluinos with masses below about $275 \mathrm{GeV} / \mathrm{c}^{2}$ for any choice of the SUSY parameters. For some parameter values the Tr ratmn will be sensitive to gluinos with masses up to $400 \mathrm{GeV} / \mathrm{c}^{2}$.

- The Tevatron can search for light supersymmetric top quarks in various decay modes up to about $180 \mathrm{GeV} / \mathrm{c}^{2}$ mass.

The Tevatron enjoys a window of opportunity to discover the first evidence for a highly motivated theory beyond the Standard Model, or to significantly constrain that theory. The increased luminosity available at TeV33 is necessary to exploit this opportunity during the next decade. 


\section{Intermediate Vector Boson Physics}

With very large integrated luminosities at the Tevatron, the electroweak sector sector of the SM can be probed in great detail. Our preliminary studies arrive at the following conclusions:

- With $10 \mathrm{fb}^{-1}$ it should be possible to measure the mass of the $W$ boson with a precision of at least $30 \mathrm{MeV} / \mathrm{c}^{2}$, and $20 \mathrm{MeV} / \mathrm{c}^{2}$ may well be within reach. This is about a factor of 2 better than what one expects for LEP II. With a precision of $20 \mathrm{MeV} / \mathrm{c}^{2}\left(30 \mathrm{MeV} / \mathrm{c}^{2}\right)$ for the $W$ mass, and $2 \mathrm{GeV} / \mathrm{c}^{2}$ for the top quark mass, the Higgs boson mass can be predicted with an uncertainty of about $40 \%(50 \%)$ of itself. This prediction may be very useful for direct Higgs searches at the Tevatron, LHC, or NLC, and comparison with the results of a direct search may constitute an ultimate test of the SM.

- The $W$ width can be measured with an uncertainty of about $15 \mathrm{MeV}$. This is an improvement of almost one order of magnitude of the current uncertainty. At LEP II $\Gamma_{W}$ can only be measured with a precision of a few hundred $\mathrm{MeV}$.

- The $W$ charge asymmetry will be a very powerful tool in constraining the parton distribution functions. In many processes the error in the parton distribution functions currently constitutes a major source of uncertainty. The forward backward asymmetry, $A_{F B}$ in $Z$ boson decays provides a useful cross check on the Higgs boson mass extracted from the $W$ mass measurement.

- With $10 \mathrm{fb}^{-1}$, the $W W V$ and $Z_{\gamma} V, V=\gamma, Z$, vertices can be determined with a precision of $\mathcal{O}(10 \%)$ and $\mathcal{O}\left(10^{-2}-10^{-3}\right)$, respectively, at the Tevatron. The expected accuracy for the $W W V$ couplings is comparable or better than that of LEP II. However, since the methods used to extract limits on anomalous couplings at the two colliders are different, data from the Tevatron and LEP II yield complementary information. Tevation experiments will be able to place limits on the $Z_{\gamma} V$ couplings which are up to a factor 100 better than those which can be achieved at LEP II. At the $\mathrm{LHC}$, with $100 \mathrm{fb}^{-1}$, it will be possible to place limits on anomalous $W W V$ and $Z \gamma V$ couplings which are a factor 3 to 100 better than those one can expect for the Tevatron with $10 \mathrm{fb}^{-1}$.

- The Tevatron offers a unique chance to search for the SM "radiation zero" in $W \gamma$ production, which provides an additional test of the gauge theory nature of the SM. At the LHC, due to the large $q g$ luminosity, QCD corrections obscure the dip in the photon lepton rapidity difference distribution which is caused by the radiation zero. This is not the case at Tevatron energies. Currently, the experimental results are statistically 
limited. With integrated luminosities of $2 \mathrm{fb}^{-1}$ or more, it should be possible to conclusively establish the existence of the radiation zero.

- With an integrated luminosity of $10 \mathrm{fb}^{-1}$, limits on the branching ratios of rare $W$ decays of $\mathcal{O}\left(10^{-5}\right)$ to $\mathcal{O}\left(10^{-7}\right)$ can be obtained. $W$ decays into two pseudoscalar mesons offer an opportunity to probe meson decay form factors at a very high momentum transfer where these form factors have not been tested so far.

- The Tevatron offers a unique opportunity to search for $C P$ violation in $W$ boson production and decay since it collides protons and antiprotons, ie. the initial state is a $C P$ eigenstate. The extremely large number of $W$ boson events expected at a superluminous Tevatron will make it possible to search for small $C P$-violating contributions to $W$ boson production, at the level of $\mathcal{O}\left(10^{-3}-10^{-4}\right)$.

- An integrated luminosity of $10 \mathrm{fb}^{-1}$, will produce a sufficient number of $W \gamma \gamma, Z \gamma \gamma$ and $W W \gamma$ events to extract direct information on the quartic gauge boson couplings.

\section{CONCLUSIONS}

As previously noted, we believe that this work is not complete. We have shown that a future path of increasing luminosity at the Tevatron will lead to a full program of measurements in Top, IVB, Higgs, SUSY, and Exotic physics. Our general conclusions are as follows:

1. Fermilab will be the top factory for many years. As with other heavy quarks, the top may be entering the first of many decades of serious scrutiny. U.S. HEP program planning should recognise this major scientific opportunity.

2. Our study confirms recent theoretical speculation that there is a luminosity threshold for the detection of a light Higgs boson at the Tevatron. The most promising single detection technique relies on detailed understanding of b-tagging and the "W + flavor" backgrounds, and is therefore a natural complement and extension of the top physics program.

(a) the presence of the detection threshold and its value be confirmed in more detailed simulation, including b-tagging in the presence of multiple interactions.

(b) a Tevatron strategy for crossing the luminosity threshold be developed and implemented.

3. The Tevatron program can either discover SUSY or significantly constrain a large fraction of current theoretical prejudice. The actual sensitivity 
and discovery potential for supersymmetric states at the Tevatron deserves significantly more study.

4. High instantaneous luminosity conditions need to be understood better with perhaps both simulation and actual detector research and development. This is especially true for the top and Higgs studies. Will it blind the current detectors? If so, how? We urge the Laboratory to initiate an active program to investigate these questions and to engage the high energy physics community in the effort. We believe the effort will benefit from computing, $R \& D$, and possibly even test beam resources.

5. There may be significant luminosity capability beyond the "classic" Main Injector scenario during Run II. If such incremental increases in peak luminosity cannot be handled by the detectors, could this capability be channeled into a significant increase in the useful longevity of $>10^{32}$ stores? After all, integrated luminosity is the key.

6. The physics overall is tantalizing, and we believe that waiting for the LHC is neither cost effective nor prudent. The Laboratory and the experimental collaborations should make every effort to maximize the physics return of the Tevatron. This implies the need for an overall plan for the long term Tevatron Program including the accelerator, the detectors, and physics simulation.

\section{ACKNOWLEDGEMENTS}

I acknowledge the dedication and work of the Tev2000 authors, and I thank the organizers of this conference for a stimulating survey of our future.

This work is supported in part by the U.S. Department of Energy under Grant DE-FG02-95ER40899.

\section{REFERENCES}

1. "Future Electroweak Physics at the Tevatron", Report of the Tev2000 Working Group, Fermilab-Pub-96/046, 1996.

2. F. Abe et al., Phys. Rev. Lett. 74, 2626 (1995).

3. E. Laenen, private communication.

4. F. Abe et al., Phys. Rev. D. 50, 2966, 1994.

5. U. Heintz, "Top Mass Analysis of Dilepton events," DØ Note 2658.

6. P. Azzi et al., "Evidence for te production in the All-hadronic Channel", CDF Internal Note 3187, June, 1995.

7. J. Incandela, "Using the B Decay Length Distribution in Top Events to Measure $\mathrm{m}_{\mathrm{t}}$, CDF Internal Note 1921, December 1992. 
8. L. Orr, T. Stelzer, and W.J. Stirling, University of Durham Preprint DTP/94/112, University of Rochester Preprint UR-1397; L. Orr and W.J. Stirling, University of Durham Preprint DTP/94/60, University of Rochester Preprint UR-1365; S. Mrenna and C.-P. Yuan, Phys. Rev. D. 46, 1007-1021, 1992; Yu. L. Dokshitzer, V.A. Khoze and S.I. Troyan, Proceedings of the 6th International Conference on Physics in Collision, Singapore 1987; G. Marchesini and B.R. Webber Nucl.Phys. B330, 261-283.

9. C.T. Hill, "Topcolor Assisted Technicolor", Fermilab-Pub-94/395-T.

10. P. Cho and E.H. Simmons, Phys. Rev.D51, 2360, (1995). D. Atwood, A. Kagan and T. Rizzo, "Constraining Anomalous Top Quark Couplings at the Tevatron", SLAC-PUB-6580, July, 1994.

11. S. Dawson, Nucl. Phys. B249, 42 (1985); S.S.D. Willenbrock and D.A. Dicus, Phys. Rev. D 34, 155 (1986); S. Dawson and S.S.D. Willenbrock, Nucl. Phys. B284, 449 (1987); C.-P. Yuan, Phys. Rev. D 41, 42 (1990); S. Cortese and R. Petronzio, Phys. Lett. B253, 494 (1991); G.V. Jikia and S.R. Slabospitsky, Phys. Lett B295, 136 (1992), R.K. Ellis and S. Parke, Phys. Rev. D 46, 3785 (1992); G. Bordes and B. van Eijk, Z. Phys. C57, 81 (1993); G. Bordes and B. van Eijk, Nucl. Phys. B435, 23 (1995): T. Stelzer and S. Willenbrock, Phys. Lett. B357, 125-130 (1995).

12. A.P. Heinson, A.S. Belyaev and E.E. Boos, "Electroweak top quark production at the Fermilab Tevatron", in the proceedings of the Workshop on Physics of the Top Quark, Iowa State University, Ames, Iowa, May 1995, hep-ph/9509274.

13. E. Malkawi, C.-P. Yuan, "A Global Analysis of the Top Quark Couplings to Gauge Bosons," Phys. Rev. D. 50, 4462, 1994.

14. A.P. Heinson and P. Baringer, DØ Note 2777 (1995).

15. H. Weerts, private communication.

16. C. Hill, Topcolor Assisted Technicolor, Fermilab-Pub-94/395-T

17. K. Lane, Top Quarks and Flavor Physics, BUHEP-95-2.

18. I. Bigi et al., Phys Lett. 181B, 157, (1986).

19. For example, see G. Kane, C.-P. Yuan, and D. Ladinsky, Phys. Rev. D. 45, 124, 1992; D. Atwood, A. Aeppli, and A. Soni, Phys. Rev. Lett 69, 2754, 1992; R.S. Chivukula, S.B. Selipsky, E.H. Simmons, Phys. Rev. Lett 69, 575, 1992; M.Peskin and P. Zerwas, talks presented at the First International Workshop on Physics and Experiments at a Linear e+e-Collider, Saariselka, Finland, September 1991; D. Carlson, E. Malkawi, C.-P. Yuan, Phys. Lett. B337, 145, 1994; K. Fujikawa and A. Yamada, Phys. Rev. Lett 49, 5890, 1994.

20. G. Kane, C.-P. Yuan, and D. Ladinsky, Phys. Rev. D. 45, 124, 1992.

21. S. Parke, Summary of Top Quark Physics, FERMILAB -Conf-94/322-T. Presented at DPF'94, University of New Mexico, Albuquerque, NM, August 2-6, 1994.

22. K. Fuji, "Top Physics at Future $\mathrm{e}^{+} \mathrm{e}^{-}$Colliders, Experimental Aspects", First International Workshop on Physics and Experiments at a Linear e+e-Collider, Saariselka, Finland, September 1991;

23. P. Igo-Kemenes, M. Martinez, R. Miquel, and S. Orteau in Second International Workshop on Physics and Experiments at a Linear e+e-Collider, Waikoloa, 
HI, April 1993.

24. $\mathrm{P}$ Igo-Kemenes in Second International Workshop on Physics and Experıments at a Linear ete-Collider, Waikoloa, HI, April 1993.

25. G. Bagliesı at., Top Quark Physics, Experimental Aspects, in First International Workshop on Physics and Experzments at a Linear e+e-Collider, Saariselka, Finland, September 1991.

26. M. Peskın at, Physics Issues for the Next Linear Collider in First Internatzonal Workshop on Physics and Experiments at a Linear e+e-Collider, Saarıelka, Finland, September 1991.

27 G. A. Ladinsky and C.-P Yuan, in Second International Workshop on Physics and Experzments at a Linear e+e-Collider, Waikoloa, HI, April 1993.

28. See for example D Froldevaux, Top Quark Physics at LHC/SSC CERN/PPE/93-148 